\title{
Intrinsic sound of anti-de Sitter manifolds
}

Toshiyuki Kobayashi

\begin{abstract}
As is well-known for compact Riemann surfaces, eigenvalues of the Laplacian are distributed discretely and most of eigenvalues vary viewed as functions on the Teichmüller space. We discuss a new feature in the Lorentzian geometry, or more generally, in pseudo-Riemannian geometry. One of the distinguished features is that $L^{2}$-eigenvalues of the Laplacian may be distributed densely in $\mathbb{R}$ in pseudo-Riemannian geometry. For three-dimensional anti-de Sitter manifolds, we also explain another feature proved in joint with F. Kassel [Adv. Math. 2016] that there exist countably many $L^{2}$-eigenvalues of the Laplacian that are stable under any small deformation of anti-de Sitter structure. Partially supported by Grant-in-Aid for Scientific Research (A) (25247006), Japan Society for the Promotion of Science. Keywords and phrases: Laplacian, locally symmetric space, Lorentzian manifold, spectral analysis, Clifford-Klein form, reductive group, discontinuous group 2010
\end{abstract}

MSC: Primary 22E40, 22E46, 58J50: Secondary 11F72, 53C35

\section{Introduction}

Our "common sense" for music instruments says:

"shorter strings produce a higher pitch than longer strings",

"thinner strings produce a higher pitch than thicker strings".

Let us try to "hear the sound of pseudo-Riemannian locally symmetric spaces". Contrary to our "common sense" in the Riemannian world, we find a phenomenon that compact three-dimensional anti-de Sitter manifolds have "intrinsic sound"

Toshiyuki Kobayashi

Kavli IPMU and Graduate School of Mathematical Sciences, The University of Tokyo, e-mail:

toshi@ms.u-tokyo.ac.jp 
which is stable under any small deformation. This is formulated in the framework of spectral analysis of anti-de Sitter manifolds, or more generally, of pseudoRiemannian locally symmetric spaces $X_{\Gamma}$. In this article, we give a flavor of this new topic by comparing it with the flat case and the Riemannian case.

To explain briefly the subject, let $X$ be a pseudo-Riemannian manifold, and $\Gamma$ a discrete isometry group acting properly discontinuously and freely on $X$. Then the quotient space $X_{\Gamma}:=\Gamma \backslash X$ carries a pseudo-Riemannian manifold structure such that the covering map $X \rightarrow X_{\Gamma}$ is isometric. We are particularly interested in the case where $X_{\Gamma}$ is a pseudo-Riemannian locally symmetric space, see Section 3.2

Problems we have in mind are symbolized in the following diagram:

\begin{tabular}{c|c|c} 
& existence problem & deformation $v . s$. rigidity \\
\hline Geometry & $\begin{array}{c}\text { Does cocompact } \Gamma \text { exist? } \\
\text { (Section 4.1) }\end{array}$ & $\begin{array}{c}\text { Higher Teichmüller theory } v . s . \text { rigidity theorem } \\
\text { (Section 4.2 }\end{array}$ \\
\hline Analysis & $\begin{array}{c}\text { Does } L^{2} \text {-spectrum exist? } \\
\text { (Problem A) }\end{array}$ & $\begin{array}{c}\text { Whether } L^{2} \text {-eigenvalues vary or not } \\
\text { (Problem B) }\end{array}$
\end{tabular}

\section{A program}

In [5, 6, 12] we initiated the study of "spectral analysis on pseudo-Riemannian locally symmetric spaces" with focus on the following two problems:

Problem A Construct eigenfunctions of the Laplacian $\Delta_{X_{\Gamma}}$ on $X_{\Gamma}$. Does there exist a nonzero $L^{2}$-eigenfunction?

Problem B Understand the behaviour of $L^{2}$-eigenvalues of the Laplacian $\Delta_{X_{\Gamma}}$ on $X_{\Gamma}$ under small deformation of $\Gamma$ inside $G$.

Even when $X_{\Gamma}$ is compact, the existence of countably many $L^{2}$-eigenvalues is already nontrivial because the Laplacian $\Delta_{X_{\Gamma}}$ is not elliptic in our setting. We shall discuss in Section 2.2 for further difficulties concerning Problems $\mathrm{A}$ and B when $X_{\Gamma}$ is non-Riemannian.

We may extend these problems by considering joint eigenfunctions for "invariant differential operators" on $X_{\Gamma}$ rather than the single operator $\Delta_{X_{\Gamma}}$. Here by "invariant differential operators on $X_{\Gamma}$ " we mean differential operators that are induced from $G$-invariant ones on $X=G / H$. In Section 7, we discuss Problems @ and B in this general formulation based on the recent joint work [6, 7] with F. Kassel. 


\subsection{Known results}

Spectral analysis on a pseudo-Riemannian locally symmetric space $X_{\Gamma}=\Gamma \backslash X=$ $\Gamma \backslash G / H$ is already deep and difficult in the following special cases:

1) (noncommutative harmonic analysis on $G / H) \Gamma=\{e\}$.

In this case, the group $G$ acts unitarily on the Hilbert space $L^{2}\left(X_{\Gamma}\right)=L^{2}(X)$ by translation $f(\cdot) \mapsto f\left(g^{-1} \cdot\right)$, and the irreducible decomposition of $L^{2}(X)$ (Plancherel-type formula) is essentially equivalent to the spectral analysis of $G$-invariant differential operators when $X$ is a semisimple symmetric space. Noncommutative harmonic analysis on semisimple symmetric spaces $X$ has been developed extensively by the work of Helgason, Flensted-Jensen, MatsukiOshima-Sekiguchi, Delorme, van den Ban-Schlichtkrull among others as a generalization of Harish-Chandra's earlier work on the regular representation $L^{2}(G)$ for group manifolds.

2) (automorphic forms) $H$ is compact and $\Gamma$ is arithmetic.

If $H$ is a maximal compact subgroup of $G$, then $X_{\Gamma}=\Gamma \backslash G / H$ is a Riemannian locally symmetric space and the Laplacian $\Delta_{X_{\Gamma}}$ is an elliptic differential operator. Then there exist infinitely many $L^{2}$-eigenvalues of $\Delta_{X_{\Gamma}}$ if $X_{\Gamma}$ is compact by the general theory for compact Riemannian manifolds (see Fact 1 ). If furthermore $\Gamma$ is irreducible, then Weil's local rigidity theorem [18] states that nontrivial deformations exist only when $X$ is the hyperbolic plane $S L(2, \mathbb{R}) / S O(2)$, in which case compact quotients $X_{\Gamma}$ have a classically-known deformation space modulo conjugation, i.e., their Teichmüller space. Viewed as a function on the Teichmüller space, $L^{2}$-eigenvalues vary analytically [1, 20], see Fact 11 .

Spectral analysis on $X_{\Gamma}$ is closely related to the theory of automorphic forms in the Archimedian place if $\Gamma$ is an arithmetic subgroup.

3) (abelian case) $G=\mathbb{R}^{p+q}$ with $H=\{0\}$ and $\Gamma=\mathbb{Z}^{p+q}$.

We equip $X=G / H$ with the standard flat pseudo-Riemannian structure of signature $(p, q)$ (see Example 11). In this case, $G$ is abelian, but $X=G / H$ is nonRiemannian. This is seemingly easy, however, spectral analysis on the $(p+q)$ torus $\mathbb{R}^{p+q} / \mathbb{Z}^{p+q}$ is much involved, as we shall observe a connection with Oppenheim's conjecture (see Section 5.2).

\subsection{Difficulties in the new settings}

If we try to attack a problem of spectral analysis on $\Gamma \backslash G / H$ in the more general case where $H$ is noncompact and $\Gamma$ is infinite, then new difficulties may arise from several points of view:

(1) Geometry. The $G$-invariant pseudo-Riemannian structure on $X=G / H$ is not Riemannian anymore, and discrete groups of isometries of $X$ do not always act properly discontinuously on such $X$. 
(2) Analysis. The Laplacian $\Delta_{X}$ on $X_{\Gamma}$ is not an elliptic differential operator. Furthermore, it is not clear if $\Delta_{X}$ has a self-adjoint extension on $L^{2}\left(X_{\Gamma}\right)$.

(3) Representation theory. If $\Gamma$ acts properly discontinuously on $X=G / H$ with $H$ noncompact, then the volume of $\Gamma \backslash G$ is infinite, and the regular representation $L^{2}(\Gamma \backslash G)$ may have infinite multiplicities. In turn, the group $G$ may not have a good control of functions on $\Gamma \backslash G$. Moreover $L^{2}\left(X_{\Gamma}\right)$ is not a subspace of $L^{2}(\Gamma \backslash G)$ because $H$ is noncompact. All these observations suggest that an application of the representation theory of $L^{2}(\Gamma \backslash G)$ to spectral analysis on $X_{\Gamma}$ is rather limited when $H$ is noncompact.

Point (1) creates some underlying difficulty to Problem B we need to consider locally symmetric spaces $X_{\Gamma}$ for which proper discontinuity of the action of $\Gamma$ on $X$ is preserved under small deformations of $\Gamma$ in $G$. This is nontrivial. This question was first studied by the author [9, 11]. See [4] for further study. An interesting aspect of the case of noncompact $H$ is that there are more examples where nontrivial deformations of compact quotients exist than for compact $H$ ( $c f$. Weil's local rigidity theorem [18]). Perspectives from Point (1) will be discussed in Section 4

Point (2) makes Problem Anontrivial. It is not clear if the following well-known properties in the Riemannian case holds in our setting in the pseudo-Riemannian case.

Fact 1 Suppose $M$ is a compact Riemannian manifold.

(1) The Laplacian $\Delta_{M}$ extends to a self-adjoint operator on $L^{2}(M)$.

(2) There exist infinitely many $L^{2}$-eigenvalues of $\Delta_{M}$.

(3) An eigenfunction of $\Delta_{M}$ is infinitely differentiable.

(4) Each eigenspace of $\Delta_{M}$ is finite-dimensional.

(5) The set of $L^{2}$-eigenvalues is discrete in $\mathbb{R}$.

Remark 1. We shall see that the third to fifth properties of Fact 1 may fail in the pseudo-Riemannian case, e.g., Example 6 for (3) and (4), and $M=\mathbb{R}^{2,1} / \mathbb{Z}^{3}$ (Theorem 7) for (5).

In spite of these difficulties, we wish to reveal a mystery of spectral analysis of pseudo-Riemannian locally homogeneous spaces $X_{\Gamma}=\Gamma \backslash G / H$. We shall discuss self-adjoint extension of the Laplacian in the pseudo-Riemannian setting in Theorem 13 , and the existence of countable many $L^{2}$-eigenvalues in Theorems 8,12 and 13

\section{Pseudo-Riemannian manifold}

\subsection{Laplacian on pseudo-Riemannian manifolds}

A pseudo-Riemannian manifold $M$ is a smooth manifold endowed with a smooth, nondegenerate, symmetric bilinear tensor $g$ of signature $(p, q)$ for some $p, q \in \mathbb{N}$. 
$(M, g)$ is a Riemannian manifold if $q=0$, and is a Lorentzian manifold if $q=1$. The metric tensor $g$ induces a Radon measure $d \mu$ on $X$, and the divergence div. Then the Laplacian

$$
\Delta_{M}:=\operatorname{div} \operatorname{grad},
$$

is a differential operator of second order which is a symmetric operator on the Hilbert space $L^{2}(X, d \mu)$.

Example 1. Let $(M, g)$ be the standard flat pseudo-Riemannian manifold:

$$
\mathbb{R}^{p, q}:=\left(\mathbb{R}^{p+q}, d x_{1}^{2}+\cdots+d x_{p}^{2}-d x_{p+1}^{2}-\cdots-d x_{p+q}^{2}\right) .
$$

Then the Laplacian takes the form

$$
\Delta_{\mathbb{R}^{p, q}}=\frac{\partial^{2}}{\partial x_{1}^{2}}+\cdots+\frac{\partial^{2}}{\partial x_{p}^{2}}-\frac{\partial^{2}}{\partial x_{p+1}^{2}}-\cdots-\frac{\partial^{2}}{\partial x_{p+q}^{2}} .
$$

In general, $\Delta_{M}$ is an elliptic differential operator if $(M, g)$ is Riemannian, and is a hyperbolic operator if $(M, g)$ is Lorentzian.

\subsection{Homogeneous pseudo-Riemannian manifolds}

A typical example of pseudo-Riemannian manifolds $X$ with "large" isometry groups is semisimple symmetric spaces, for which the infinitesimal classification was accomplished by M. Berger in 1950s. In this case, $X$ is given as a homogeneous space $G / H$ where $G$ is a semisimple Lie group and $H$ is an open subgroup of the fixed point group $G^{\sigma}=\{g \in G: \sigma g=g\}$ for some involutive automorphism $\sigma$ of $G$. In particular, $G \supset H$ are a pair of reductive Lie groups.

More generally, we say $G / H$ is a reductive homogeneous space if $G \supset H$ are a pair of real reductive algebraic groups. Then we have the following:

Proposition 1. Any reductive homogeneous space $X=G / H$ carries a pseudoRiemannian structure such that $G$ acts on $X$ by isometries.

Proof. By a theorem of Mostow, we can take a Cartan involution $\theta$ of $G$ such that $\theta H=H$. Then $K:=G^{\theta}$ is a maximal compact subgroup of $G$, and $H \cap K$ is that of $H$. Let $\mathfrak{g}=\mathfrak{k}+\mathfrak{p}$ be the corresponding Cartan decomposition of the Lie algebra $\mathfrak{g}$ of $G$. Take an $\operatorname{Ad}(G)$-invariant nondegenerate symmetric bilinear form $\langle$,$\rangle on \mathfrak{g}$ such that $\left.\langle\rangle\right|_{,\mathfrak{k} \times \mathfrak{k}}$ is negative definite, $\left.\langle\rangle\right|_{,\mathfrak{p} \times \mathfrak{p}}$ is positive definite, and $\mathfrak{k}$ and $\mathfrak{p}$ are orthogonal to each other. (If $G$ is semisimple, then we may take $\langle$,$\rangle to be the Killing$ form of $\mathfrak{g}$. )

Since $\theta H=H$, the Lie algebra $\mathfrak{h}$ of $H$ is decomposed into a direct sum $\mathfrak{h}=(\mathfrak{h} \cap$ $\mathfrak{k})+(\mathfrak{h} \cap \mathfrak{p})$, and therefore the bilinear form $\langle$,$\rangle is non-degenerate when restricted$ to $\mathfrak{h}$. Then $\langle$,$\rangle induces an \operatorname{Ad}(H)$-invariant nondegenerate symmetric bilinear form $\langle,\rangle_{\mathfrak{g} / \mathfrak{h}}$ on the quotient space $\mathfrak{g} / \mathfrak{h}$, with which we identify the tangent space $T_{o}(G / H)$ 
at the origin $o=e H \in G / H$. Since the bilinear form $\langle,\rangle_{\mathfrak{g} / \mathfrak{h}}$ is $\operatorname{Ad}(H)$-invariant, the left translation of this form is well-defined and gives a pseudo-Riemannian structure $g$ on $G / H$ of signature $(\operatorname{dim} \mathfrak{p} / \mathfrak{h} \cap \mathfrak{p}, \operatorname{dim} \mathfrak{k} / \mathfrak{h} \cap \mathfrak{k})$. By the construction, the group $G$ acts on the pseudo-Riemannian manifold $(G / H, g)$ by isometries.

\subsection{Pseudo-Riemannian manifolds with constant curvature, Anti-de Sitter manifolds}

Let $Q_{p, q}(x):=x_{1}^{2}+\cdots+x_{p}^{2}-x_{p+1}^{2}-\cdots-x_{p+q}^{2}$ be a quadratic form on $\mathbb{R}^{p+q}$ of signature $(p, q)$, and we denote by $O(p, q)$ the indefinite orthogonal group preserving the form $Q_{p, q}$. We define two hypersurfaces $M_{ \pm}^{p, q}$ in $\mathbb{R}^{p+q}$ by

$$
M_{ \pm}^{p, q}:=\left\{x \in \mathbb{R}^{p+q}: Q_{p, q}(x)= \pm 1\right\} .
$$

By switching $p$ and $q$, we have an obvious diffeomorphism

$$
M_{+}^{p, q} \simeq M_{-}^{q, p}
$$

The flat pseudo-Riemannian structure $\mathbb{R}^{p, q}$ (Example1) induces a pseudo-Riemannian structure on the hypersurface $M_{+}^{p, q}$ of signature $(p-1, q)$ with constant curvature 1, and that on $M_{-}^{p, q}$ of signature $(p, q-1)$ with constant curvature -1 .

The natural action of the group $O(p, q)$ on $\mathbb{R}^{p, q}$ induces an isometric and transitive action on the hypersurfaces $M_{ \pm}^{p, q}$, and thus they are expressed as homogeneous spaces:

$$
M_{+}^{p, q} \simeq O(p, q) / O(p-1, q), \quad M_{-}^{p, q} \simeq O(p, q) / O(p, q-1),
$$

giving examples of pseudo-Riemannian homogeneous spaces as in Proposition 1

The anti-de Sitter space $\mathrm{AdS}^{n}=M_{-}^{n-1,2}$ is a model space for $n$-dimensional Lorentzian manifolds of constant negative sectional curvature, or anti-de Sitter $n$ manifolds. This is a Lorentzian analogue of the real hyperbolic space $H^{n}$. For the convenience of the reader, we list model spaces of Riemannian and Lorentzian manifolds with constant positive, zero, and negative curvatures.

Riemannian manifolds with constant curvature:

$$
\begin{array}{ll}
S^{n}=M_{+}^{n+1,0} \simeq O(n+1) / O(n) & : \text { standard sphere, } \\
\mathbb{R}^{n} & : \text { Euclidean space, } \\
H^{n}=M_{-}^{n, 1} \simeq O(1, n) / O(n) & : \text { hyperbolic space, }
\end{array}
$$

Lorentzian manifolds with constant curvature: 


$$
\begin{aligned}
& \mathrm{d} \mathrm{S}^{n}=M_{+}^{n, 1} \simeq O(n, 1) / O(n-1,1): \text { de Sitter space } \\
& \mathbb{R}^{n-1,1}: \text { Minkowski space, } \\
& \mathrm{AdS}^{n}=M_{-}^{n-1,2} \simeq O(2, n-1) / O(1, n-1): \text { anti-de Sitter space }
\end{aligned}
$$

\section{Discontinuous groups for pseudo-Riemannian manifolds}

\subsection{Existence problem of compact Clifford-Klein forms}

Let $H$ be a closed subgroup of a Lie group $G$, and $X=G / H$, and $\Gamma$ a discrete subgroup of $G$. If $H$ is compact, then the double coset space $\Gamma \backslash G / H$ becomes a $C^{\infty}$-manifold for any torsion-free discrete subgroup $\Gamma$ of $G$. However, we have to be careful for noncompact $H$, because not all discrete subgroups acts properly discontinuously on $G / H$, and $\Gamma \backslash G / H$ may not be Hausdorff in the quotient topology. We illustrate this feature by two general results:

Fact 2(1) (Moore's ergodicity theorem [15]) Let $G$ be a simple Lie group, and $\Gamma$ a lattice. Then $\Gamma$ acts ergodically on $G / H$ for any noncompact closed subgroup $H$. In particular, $\Gamma \backslash G / H$ is non-Hausdorff.

(2) (Calabi-Markus phenomenon ([2, 8])) Let $G$ be a reductive Lie group, and $\Gamma$ an infinite discrete subgroup. Then $\Gamma \backslash G / H$ is non-Hausdorff for any reductive subgroup $H$ with $\operatorname{rank}_{\mathbb{R}} G=\operatorname{rank}_{\mathbb{R}} H$.

In fact, determining which groups act properly discontinuously on reductive homogeneous spaces $G / H$ is a delicate problem, which was first considered in full generality by the author; we refer to [13, Section 3.2] for a survey.

Suppose now a discrete subgroup $\Gamma$ acts properly discontinuously and freely on $X=G / H$. Then the quotient space

$$
X_{\Gamma}:=\Gamma \backslash X \simeq \Gamma \backslash G / H
$$

carries a $C^{\infty}$-manifold structure such that the quotient map $p: X \rightarrow X_{\Gamma}$ is a covering, through which $X_{\Gamma}$ inherits any $G$-invariant local geometric structure on $X$. We say $\Gamma$ is a discontinuous group for $X$ and $X_{\Gamma}$ is a Clifford-Klein form of $X=G / H$.

Example 2.(1) If $X=G / H$ is a reductive homogeneous space, then any CliffordKlein form $X_{\Gamma}$ carries a pseudo-Riemannian structure by Proposition 1.

(2) If $X=G / H$ is a semisimple symmetric space, then any Clifford-Klein form $X_{\Gamma}=$ $\Gamma \backslash G / H$ is a pseudo-Riemannian locally symmetric space, namely, the (local) geodesic symmetry at every $p \in X_{\Gamma}$ with respect to the Levi-Civita connection is locally isometric.

By space forms, we mean pseudo-Riemannian manifolds of constant sectional curvature. They are examples of pseudo-Riemannian locally symmetric spaces. For simplicity, we shall assume that they are geodesically complete. 
Example 3. Clifford-Klein forms of $M_{+}^{p+1, q}=O(p+1, q) / O(p, q)$ (respectively, $\left.M_{-}^{p, q+1}=O(p, q+1) / O(p, q)\right)$ are pseudo-Riemannian space forms of signature $(p, q)$ with positive (respectively, negative) curvature. Conversely, any (geodesically complete) pseudo-Riemannian space form of signature $(p, q)$ is of this form as far as $p \neq 1$ for positive curvature or $q \neq 1$ for negative curvature.

A general question for reductive homogeneous spaces $G / H$ is:

Question 1. Does compact Clifford-Klein forms of $G / H$ exist?

or equivalently,

Question 2. Does there exist a discrete subgroup $\Gamma$ of $G$ acting cocompactly and properly discontinuously on $G / H$ ?

This question has an affirmative answer if $H$ is compact by a theorem of Borel. In the general setting where $H$ is noncompact, the question relates with a "global theory" of pseudo-Riemannian geometry: how local pseudo-Riemannian homogeneous structure affects the global nature of manifolds? A classic example is space form problem which asks the global properties (e.g. compactness, volume, fundamental groups, etc.) of a pseudo-Riemannian manifold of constant curvature (local property). The study of discontinuous groups for $M_{+}^{p+1, q}$ and $M_{-}^{p, q+1}$ shows the following results in pseudo-Riemannian space forms of signature $(p, q)$ :

Fact 3 Space forms of positive curvature are

(1) always closed if $q=0$, i.e., sphere geometry in the Riemannian case;

(2) never closed if $p \geq q>0$, in particular, if $q=1$ (de Sitter geometry in the Lorentzian case [2]7).

The phenomenon in the second statement is called the Calabi-Markus phenomenon (see Fact 2 (2) in the general setting).

Fact 4 Compact space forms of negative curvature exist

(1) for all dimensions if $q=0$, i.e., hyperbolic geometry in the Riemannian case;

(2) for odd dimensions if $q=1$, i.e., anti-de Sitter geometry in the Lorentzian case;

(3) for $(p, q)=(4 m, 3)(m \in \mathbb{N})$ or $(8,7)$.

See [13, Section 4] for the survey of the space form problem in pseudo-Riemannian geometry and also of Question 1 for more general $G / H$.

A large and important class of Clifford-Klein forms $X_{\Gamma}$ of a reductive homogeneous space $X=G / H$ is constructed as follows (see [8]).

Definition 1. A quotient $X_{\Gamma}=\Gamma \backslash X$ of $X$ by a discrete subgroup $\Gamma$ of $G$ is called standard if $\Gamma$ is contained in some reductive subgroup $L$ of $G$ acting properly on $X$.

If a subgroup $L$ acts properly on $G / H$, then any discrete subgroup of $\Gamma$ acts properly discontinuously on $G / H$. A handy criterion for the triple $(G, H, L)$ of reductive groups such that $L$ acts properly on $G / H$ is proved in [8], as we shall recall below. 
Let $G=K \exp \overline{\mathfrak{a}_{+}} K$ be a Cartan decomposition, where $\mathfrak{a}$ is a maximal abelian subspace of $\mathfrak{p}$ and $\overline{\mathfrak{a}}_{+}$is the dominant Weyl chamber with respect to a fixed positive system $\Sigma^{+}(\mathfrak{g}, \mathfrak{a})$. This defines a map $\mu: G \rightarrow \overline{\mathfrak{a}_{+}}$(Cartan projection) by

$$
\mu\left(k_{1} e^{X} k_{2}\right)=X \quad \text { for } k_{1}, k_{2} \in K \text { and } X \in \mathfrak{a} .
$$

It is continuous, proper and surjective. If $H$ is a reductive subgroup, then there exists $g \in G$ such that $\mu\left(\mathrm{gHg}^{-1}\right)$ is given by the intersection of $\overline{\mathfrak{a}}_{+}$with a subspace of dimension $\operatorname{rank}_{\mathbb{R}} H$. By an abuse of notation, we use the same $H$ instead of $g \mathrm{Hg}^{-1}$. With this convention, we have:

Properness Criterion 5 ([|8|) L acts properly on $G / H$ if and only if $\mu(L) \cap \mu(H)=$ $\{0\}$.

By taking a lattice $\Gamma$ of such $L$, we found a family of pseudo-Riemannian locally symmetric spaces $X_{\Gamma}$ in [8, 13]. The list of symmetric spaces admitting standard Clifford-Klein forms of finite volume (or compact forms) include $M_{-}^{p, q+1}=$ $O(p, q+1) / O(p, q)$ with $(p, q)$ satisfying the conditions in Fact 4 Further, by applying Properness Criterion 5] Okuda [16] gave examples of pseudo-Riemannian locally symmetric spaces $\Gamma \backslash G / H$ of infinite volume where $\Gamma$ is isomorphic to the fundamental group $\pi_{1}\left(\Sigma_{g}\right)$ of a compact Riemann surface $\Sigma_{g}$ with $g \geq 2$.

For the construction of stable spectrum on $X_{\Gamma}$ (see Theorem 10 and Theorem 12 (2) below), we introduced in [6, Section 1.6] the following concept:

Definition 2. A discrete subgroup $\Gamma$ of $G$ acts strongly properly discontinuously (or sharply) on $X=G / H$ if there exists $C, C^{\prime}>0$ such that for all $\gamma \in \Gamma$,

$$
d(\mu(\gamma), \mu(H)) \geq C\|\mu(\gamma)\|-C^{\prime}
$$

Here $d(\cdot, \cdot)$ is a distance in $\mathfrak{a}$ given by a Euclidean norm $\|\cdot\|$ which is invariant under the Weyl group of the restricted root system $\Sigma(\mathfrak{g}, \mathfrak{a})$. We say the positive number $C$ is the first sharpness constant for $\Gamma$.

If a reductive subgroup $L$ acts properly on a reductive homogeneous space $G / H$, then the action of a discrete subgroup $\Gamma$ of $L$ is strongly properly discontinuous ( 6 , Example 4.10]).

\subsection{Deformation of Clifford-Klein forms}

Let $G$ be a Lie group and $\Gamma$ a finitely generated group. We denote by $\operatorname{Hom}(\Gamma, G)$ the set of all homomorphisms of $\Gamma$ to $G$ topologized by pointwise convergence. By taking a finite set $\left\{\gamma_{1}, \cdots, \gamma_{k}\right\}$ of generators of $\Gamma$, we can identify $\operatorname{Hom}(\Gamma, G)$ as a subset of the direct product $G \times \cdots \times G$ by the inclusion:

$$
\operatorname{Hom}(\Gamma, G) \hookrightarrow G \times \cdots \times G, \quad \varphi \mapsto\left(\varphi\left(\gamma_{1}\right), \cdots, \varphi\left(\gamma_{k}\right)\right) .
$$


If $\Gamma$ is finitely presentable, then $\operatorname{Hom}(\Gamma, G)$ is realized as a real analytic variety via (1).

Suppose $G$ acts continuously on a manifold $X$. We shall take $X=G / H$ with noncompact closed subgroup $H$ later. Then not all discrete subgroups act properly discontinuously on $X$ in this general setting. The main difference of the following definition of the author [9] in the general case from that of Weil [18] is a requirement of proper discontinuity.

$$
\begin{aligned}
R(\Gamma, G ; X):= & \{\varphi \in \operatorname{Hom}(\Gamma, G): \varphi \text { is injective, } \\
& \text { and } \varphi(\Gamma) \text { acts properly discontinuously and freely on } G / H\} .
\end{aligned}
$$

Suppose now $X=G / H$ for a closed subgroup $H$. Then the double coset space $\varphi(\Gamma) \backslash G / H$ forms a family of manifolds that are locally modelled on $G / H$ with parameter $\varphi \in R(\Gamma, G ; X)$. To be more precise on "parameter", we note that the conjugation by an element of $G$ induces an automorphism of $\operatorname{Hom}(\Gamma, G)$ which leaves $R(\Gamma, G ; X)$ invariant. Taking these unessential deformations into account, we define the deformation space (generalized Teichmüller space) as the quotient set

$$
\mathscr{T}(\Gamma, G ; X):=R(\Gamma, G ; X) / G .
$$

Example 4.(1) Let $\Gamma$ be the surface group $\pi_{1}\left(\Sigma_{g}\right)$ of genus $g \geq 2, G=P S L(2, \mathbb{R})$, $X=H^{2}$ (two-dimensional hyperbolic space). Then $\mathscr{T}(\Gamma, G ; X)$ is the classical Teichmüller space, which is of dimension $6 g-6$.

(2) $G=\mathbb{R}^{n}, X=\mathbb{R}^{n}, \Gamma=\mathbb{Z}^{n}$. Then $\mathscr{T}(\Gamma, G ; X) \simeq G L(n, \mathbb{R})$ (see (4) below).

(3) $G=S O(2,2), X=\operatorname{AdS}^{3}$, and $\Gamma=\pi_{1}\left(\Sigma_{g}\right)$. Then $\mathscr{T}(\Gamma, G ; X)$ is of dimension $12 g-12$ (see [6, Section 9.2] and references therein).

Remark 2. There is a natural isometry between $X_{\varphi(\Gamma)}$ and $X_{\varphi\left(g \Gamma g^{-1}\right)}$. Hence, the set $\operatorname{Spec}_{d}\left(X_{\varphi(\Gamma)}\right)$ of $L^{2}$-eigenvalues is independent of the conjugation of $\varphi \in R(\Gamma, G ; X)$ by an element of $G$. By an abuse of notation we shall write $\operatorname{Spec}_{d}\left(X_{\varphi(\Gamma)}\right)$ for $\varphi \in$ $\mathscr{T}(\Gamma, G ; X)$ when we deal with Problem B of Section 2

\section{Spectrum on $\mathbb{R}^{p, q} / \mathbb{Z}^{p+q}$ and Oppenheim conjecture}

This section gives an elementary but inspiring observation of spectrum on flat pseudo-Riemannian manifolds.

\subsection{Spectrum of $\mathbb{R}^{p, q} / \varphi\left(\mathbb{Z}^{p+q}\right)$}

Let $G=\mathbb{R}^{n}$ and $\Gamma=\mathbb{Z}^{n}$. Then the group homomorphism $\varphi: \Gamma \rightarrow G$ is uniquely determined by the image $\varphi\left(\mathbf{e}_{j}\right)(1 \leq j \leq n)$ where $\mathbf{e}_{1}, \cdots, \mathbf{e}_{n} \in \mathbb{Z}^{n}$ are the standard 
basis, and thus we have a bijection

$$
\operatorname{Hom}(\Gamma, G) \tilde{\leftarrow} M(n, \mathbb{R}), \quad \varphi_{g} \leftarrow g
$$

by $\varphi_{g}(\mathbf{m}):=g \mathbf{m}$ for $\mathbf{m} \in \mathbb{Z}^{n}$, or equivalently, by $g=\left(\varphi_{g}\left(\mathbf{e}_{1}\right), \cdots, \varphi_{g}\left(\mathbf{e}_{n}\right)\right)$.

Let $\sigma \in \operatorname{Aut}(G)$ be defined by $\sigma(\mathbf{x}):=-\mathbf{x}$. Then $H:=G^{\sigma}=\{0\}$ and $X:=$ $G / H \simeq \mathbb{R}^{n}$ is a symmetric space. The discrete group $\Gamma$ acts properly discontinuously on $X$ via $\varphi_{g}$ if and only if $g \in G L(n, \mathbb{R})$. Moreover, since $G$ is abelian, $G$ acts trivially on $\operatorname{Hom}(\Gamma, G)$ by conjugation, and therefore the deformation space $\mathscr{T}(\Gamma, G ; X)$ identifies with $R(\Gamma, G ; X)$. Hence we have a natural bijection between the two subsets of (3):

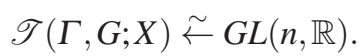

Fix $p, q \in \mathbb{N}$ such that $p+q=n$, and we endow $X \simeq \mathbb{R}^{n}$ with the standard flat indefinite metric $\mathbb{R}^{p, q}$ (see Example 11). Let us determine $\operatorname{Spec}_{d}\left(X_{\varphi_{g}(\Gamma)}\right) \simeq$ $\operatorname{Spec}_{d}\left(\mathbb{R}^{p, q} / \varphi_{g}\left(\mathbb{Z}^{n}\right)\right)$ for $g \in G L(n, \mathbb{R}) \simeq \mathscr{T}(\Gamma, G ; X)$.

For this, we define a function on $X=\mathbb{R}^{n}$ by

$$
f_{\mathbf{m}}(\mathbf{x}):=\exp \left(2 \pi \sqrt{-1}^{t} \mathbf{m} g^{-1} \mathbf{x}\right) \quad\left(\mathbf{x} \in \mathbb{R}^{n}\right)
$$

for each $\mathbf{m} \in \mathbb{Z}^{n}$ where $\mathbf{x}$ and $\mathbf{m}$ are regarded as column vectors. Clearly, $f_{\mathbf{m}}$ is $\varphi_{g}(\Gamma)$-periodic and defines a real analytic function on $X_{\varphi_{g}(\Gamma)}$. Furthermore, $f_{\mathbf{m}}$ is an eigenfunction of the Laplacian $\Delta_{\mathbb{R}}^{p, q}$ :

$$
\Delta_{\mathbb{R}^{p, q}} f_{\mathbf{m}}=-4 \pi^{2} Q_{g^{-1} I_{p, q} g^{-1}}(\mathbf{m}) f_{\mathbf{m}},
$$

where, for a symmetric matrix $S \in M(n, \mathbb{R}), Q_{S}$ denotes the quadratic form on $\mathbb{R}^{n}$ given by

$$
Q_{S}(\mathbf{y}):={ }^{t} \mathbf{y} S \mathbf{y} \quad \text { for } \mathbf{y} \in \mathbb{R}^{n} .
$$

Since $\left\{f_{\mathbf{m}}: \mathbf{m} \in \mathbb{Z}^{n}\right\}$ spans a dense subspace of $L^{2}\left(X_{\varphi_{g}(\Gamma)}\right)$, we have shown:

Proposition 2. For any $g \in G L(n, \mathbb{R}) \simeq \mathscr{T}(\Gamma, G ; X)$,

$$
\operatorname{Spec}_{d}\left(X_{\varphi_{g}(\Gamma)}\right)=\left\{-4 \pi^{2} Q_{g^{-1} I_{p, q} g^{-1}}(\mathbf{m}): \mathbf{m} \in \mathbb{Z}^{n}\right\} .
$$

Here are some observation in the $n=1,2$ cases.

Example 5. Let $n=1$ and $(p, q)=(1,0)$. Then $\operatorname{Spec}_{d}\left(X_{\varphi_{g}(\Gamma)}\right)=\left\{-4 \pi^{2} m^{2} / g^{2}: m \in\right.$ $\mathbb{Z}\}$ for $g \in \mathbb{R}^{\times} \simeq G L(1, \mathbb{R})$ by Proposition 2 . Thus the smaller the period $|g|$ is, the larger the absolute value of the eigenvalue $\left|-4 \pi^{2} \mathrm{~m}^{2} / \mathrm{g}^{2}\right|$ becomes for each fixed $m \in \mathbb{Z} \backslash\{0\}$. This is thought of as a mathematical model of a music instrument for which shorter strings produce a higher pitch than longer strings (see Introduction).

Example 6. Let $n=2$ and $(p, q)=(1,1)$. Take $g=I_{2}$, so that $\varphi_{g}(\Gamma)=\mathbb{Z}^{2}$ is the standard lattice. Then the $L^{2}$-eigenspace of the Laplacian $\Delta_{\mathbb{R}^{1,1} / \mathbb{Z}^{2}}$ for zero eigenvalue contains $W:=\left\{\psi(x-y): \psi \in L^{2}(\mathbb{R} / \mathbb{Z})\right\}$. Since $W$ is infinite-dimensional and $W \not \subset C^{\infty}\left(\mathbb{R}^{2} / \mathbb{Z}^{2}\right)$, the third and fourth statements of Fact 1 fail in this pseudoRiemannian setting. 
By the explicit description of $\operatorname{Spec}_{d}\left(X_{\varphi(\Gamma)}\right)$ for all $\varphi \in \mathscr{T}(\Gamma, G ; X)$ in Proposition 2 we can also tell the behaviour of $\operatorname{Spec}_{d}\left(X_{\varphi(\Gamma)}\right)$ under deformation of $\Gamma$ by $\varphi$. Obviously, any constant function on $X_{\varphi(\Gamma)}$ is an eigenfunction of the Laplacian $\Delta_{X_{\varphi(\Gamma)}}=\Delta_{\mathbb{R}^{p, q}} / \varphi\left(\mathbb{Z}^{p+q}\right)$ with eigenvalue zero. We see that this is the unique stable $L^{2}$-eigenvalue in the flat compact manifold:

Corollary 1 (non-existence of stable eigenvalues). Let $n=p+q$ with $p, q \in \mathbb{N}$. For any open subset $V$ of $\mathscr{T}(\Gamma, G ; X)$,

$$
\bigcap_{\varphi \in V} \operatorname{Spec}_{d}\left(X_{\varphi(\Gamma)}\right)=\{0\} .
$$

\subsection{Oppenheim's conjecture and stability of spectrum}

In 1929, Oppenheim [17] raised a question about the distribution of an indefinite quadratic forms at integral points. The following theorem, referred to as Oppenheim's conjecture, was proved by Margulis (see [14] and references therein).

Fact 6 (Oppenheim's conjecture) Suppose $n \geq 3$ and $Q$ is a real nondegenerate indefinite quadratic form in $n$ variables. Then either $Q$ is proportional to a form with integer coefficients (and thus $Q\left(\mathbb{Z}^{n}\right)$ is discrete in $\mathbb{R}$ ), or $Q\left(\mathbb{Z}^{n}\right)$ is dense in $\mathbb{R}$.

Combining this with Proposition 2 we get the following.

Theorem 7. Let $p+q=n, p \geq 2, q \geq 1, G=\mathbb{R}^{n}, X=\mathbb{R}^{p, q}$ and $\Gamma=\mathbb{Z}^{n}$. We define an open dense subset $U$ of $\mathscr{T}(\Gamma, G ; X) \simeq G L(n, \mathbb{R})$ by

$$
U:=\left\{g \in G L(n, \mathbb{R}): g^{-1} I_{p, q}{ }^{t} g^{-1} \text { is not proportional to an element of } M(n, \mathbb{Z}) .\right\}
$$

Then the set $\operatorname{Spec}_{d}\left(X_{\varphi(\Gamma)}\right)$ of $L^{2}$-eigenvalues of the Laplacian is dense in $\mathbb{R}$ if and only if $\varphi \in U$.

Thus the fifth statement of Fact 1 for compact Riemannian manifolds do fail in the pseudo-Riemannian case.

\section{Main results—-sound of anti-de Sitter manifolds}

\subsection{Intrinsic sound of anti-de Sitter manifolds}

In general, it is not clear whether the Laplacian $\Delta_{M}$ admits infinitely many $L^{2}$ eigenvalues for compact pseudo-Riemannian manifolds. For anti-de Sitter 3-manifolds, we proved in [6, Theorem 1.1]: 
Theorem 8. For any compact anti-de Sitter 3-manifold $M$, there exist infinitely many $L^{2}$-eigenvalues of the Laplacian $\Delta_{M}$.

In the abelian case, it is easy to see that compactness of $X_{\Gamma}$ is necessary for the existence of $L^{2}$-eigenvalues:

Proposition 3. Let $G=\mathbb{R}^{p+q}, X=\mathbb{R}^{p, q}, \Gamma=\mathbb{Z}^{k}$, and $\varphi \in R(\Gamma, G ; X)$. Then $\operatorname{Spec}_{d}\left(X_{\varphi(\Gamma)}\right) \neq$ $\emptyset$ if and only if $X_{\varphi(\Gamma)}$ is compact, or equivalently, $k=p+q$.

However, anti-de Sitter 3-manifolds $M$ admit infinitely many $L^{2}$-eigenvalues even when $M$ is of infinite-volume (see [6, Theorem 9.9]):

Theorem 9. For any finitely generated discrete subgroup $\Gamma$ of $G=S O(2,2)$ acting properly discontinuously and freely on $\mathrm{X}=\mathrm{AdS}^{3}$,

$$
\operatorname{Spec}_{d}\left(X_{\Gamma}\right) \supset\left\{l(l-2): l \in \mathbb{N}, l \geq 10 C^{-3}\right\}
$$

where $C \equiv C(\Gamma)$ is the first sharpness constant of $\Gamma$.

The above $L^{2}$-eigenvalues are stable in the following sense:

Theorem 10 (stable $L^{2}$-eigenvalues). Suppose that $\Gamma \subset G=S O(2,2)$ and $M=$ $\Gamma \backslash \mathrm{AdS}^{3}$ is a compact standard anti-de Sitter 3-manifold. Then there exists a neighbourhood $U \subset \operatorname{Hom}(\Gamma, G)$ of the natural inclusion with the following two properties:

$$
\begin{gathered}
U \subset R\left(\Gamma, G ; \operatorname{AdS}^{3}\right), \\
\#\left(\bigcap_{\varphi \in U} \operatorname{Spec}_{d}\left(X_{\Gamma}\right)\right)=\infty .
\end{gathered}
$$

The first geometric property (5) asserts that a small deformation of $\Gamma$ keeps proper discontinuity, which was conjectured by Goldman [3] in the $\operatorname{AdS}^{3}$ setting, and proved affirmatively in [11]. Theorem 10] was proved in [6, Corollary 9.10] in a stronger form (e.g., without assuming "standard" condition).

Figuratively speaking, Theorem [10 says that compact anti-de Sitter manifolds have "intrinsic sound" which is stable under any small deformation of the anti-de Sitter structure. This is a new phenomenon which should be in sharp contrast to the abelian case (Corollary 1) and the Riemannian case below:

Fact 11 (see [20, Theorem 5.14]) For a compact hyperbolic surface, no eigenvalue of the Laplacian above $\frac{1}{4}$ is constant on the Teichmüller space.

We end this section by raising the following question in connection with the flat case (Theorem 7):

Question 3. Suppose $M$ is a compact anti-de Sitter 3-manifold. Find a geometric condition on $M$ such that $\operatorname{Spec}_{d}(M)$ is discrete. 


\section{Perspectives and sketch of proof}

The results in the previous section for anti-de Sitter 3-manifolds can be extended to more general pseudo-Riemannian locally symmetric spaces of higher dimension:

Theorem 12 ([6, Theorem 1.5]). Let $X_{\Gamma}$ be a standard Clifford-Klein form of a semisimple symmetric space $X=G / H$ satisfying the rank condition

$$
\operatorname{rank} G / H=\operatorname{rank} K / H \cap K \text {. }
$$

Then the following holds.

(1) There exists an explicit infinite subset I of joint $L^{2}$-eigenvalues for all the differential operators on $X_{\Gamma}$ that are induced from $G$-invariant differential operators on $X$.

(2) (stable spectrum) If $\Gamma$ is contained in a simple Lie group L of real rank one acting properly on $X=G / H$, then there is a neighbourhood $V \subset \operatorname{Hom}(\Gamma, G)$ of the natural inclusion such that for any $\varphi \in V$, the action $\varphi(\Gamma)$ on $X$ is properly discontinuous and the set of joint $L^{2}$-eigenvalues on $X_{\varphi(\Gamma)}$ contains the infinite set $I$.

Remark 3. We do not require $X_{\Gamma}$ to be of finite volume in Theorem 12 .

Remark 4. It is plausible that for a general locally symmetric space $\Gamma \backslash G / H$ with $G$ reductive, no nonzero $L^{2}$-eigenvalue is stable under nontrivial small deformation unless the rank condition (7) is satisfied. For instance, suppose $\Gamma=\pi_{1}\left(\Sigma_{g}\right)$ with $g \geq 2$ and $R(\Gamma, G ; X) \neq \emptyset$. (Such semisimple symmetric space $X=G / H$ was recently classified in [16].) Then we expect the rank condition (7) is equivalent to the existence of an open subset $U$ in $R(\Gamma, G ; X)$ such that

$$
\#\left(\bigcap_{\varphi \in U} \operatorname{Spec}_{d}\left(X_{\varphi(\Gamma)}\right)\right)=\infty
$$

It should be noted that not all $L^{2}$-eigenvalues of compact anti-de Sitter manifolds are stable under small deformation of anti-de Sitter structure. In fact, we proved in [7] that there exist also countably many negative $L^{2}$-eigenvalues that are NOT stable under deformation, whereas the countably many stable $L^{2}$-eigenvalues that we constructed in Theorem 9 are all positive. More generally, we prove in [7] the following theorem that include both stable and unstable $L^{2}$-eigenvalues:

Theorem 13. Let $G$ be a reductive homogeneous space and $L$ a reductive subgroup of $G$ such that $H \cap L$ is compact. Assume that the complexification $X_{\mathbb{C}}$ is

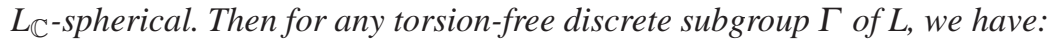

(1) the Laplacian $\Delta_{X_{\Gamma}}$ extends to a self-adjoint operator on $L^{2}\left(X_{\Gamma}\right)$;

(2) $\# \operatorname{Spec}_{d}\left(X_{\Gamma}\right)=\infty$ if $X_{\Gamma}$ is compact. 
By " $L_{\mathbb{C}}$-spherical" we mean that a Borel subgroup $L_{\mathbb{C}}$ has an open orbit in $X_{\mathbb{C}}$. In this case, a reductive subgroup $L$ acts transitively on $X$ by [10, Lemma 5.1].

Here are some examples of the setting of Theorem 13$]$ taken from [13, Corollary 3.3.7].

Table 1 1

\begin{tabular}{c|c|c|c} 
& $G$ & $H$ & $L$ \\
\hline (i) & $S O(2 n, 2)$ & $S O(2 n, 1)$ & $U(n, 1)$ \\
(ii) & $S O(2 n, 2)$ & $U(n, 1)$ & $S O(2 n, 1)$ \\
(iii) & $S U(2 n, 2)$ & $U(2 n, 1)$ & $S p(n, 1)$ \\
(iv) & $S U(2 n, 2)$ & $S p(n, 1)$ & $U(2 n, 1)$ \\
(v) & $S O(4 n, 4)$ & $S O(4 n, 3)$ & $S p(1) \times S p(n, 1)$ \\
(vi) & $S O(8,8)$ & $S O(8,7)$ & $\operatorname{Spin}(8,1)$ \\
(vii) & $S O(8, \mathbb{C})$ & $S O(7, \mathbb{C})$ & $S p i n(7,1)$ \\
(viii) & $S O(4,4)$ & $S p i n(4,3)$ & $S O(4,1) \times S O(3)$ \\
(ix) & $S O(4,3)$ & $G_{2}(\mathbb{R})$ & $S O(4,1) \times S O(2)$
\end{tabular}

Examples for Theorem 13 include Table 1 (ii) for all $n \in \mathbb{N}$, whereas we need $n \in 2 \mathbb{N}$ in Theorem 12 for the rank condition (7).

The idea of the proof for Theorem 12 is to take an average of a (nonperiodic) eigenfunction on $X$ with rapid decay at infinity over $\Gamma$-orbits as a generalization of Poincaré series. Geometric ingredients of the convergence (respectively, nonzeroness) of the generalized Poincaré series include "counting $\Gamma$-orbits" stated in Lemma11 below (respectively, the Kazhdan-Margulis theorem, $c f$. [6, Proposition 8.14]). Let $B(o, R)$ be a "pseudo-ball" of radius $R>0$ centered at the origin $o=e H \in X=G / H$, and we set

$$
N(x, R):=\#\{\gamma \in \Gamma: \gamma \cdot x \in B(o, R)\} .
$$

\section{Lemma 1 ([6, Corollary 4.7]).}

(1) If $\Gamma$ acts properly discontinuously on $X$, then $N(x, R)<\infty$ for all $x \in X$ and $R>0$.

(2) If $\Gamma$ acts strongly properly discontinuously on $X$, then there exists $A_{x}>0$ such that

$$
N(x, R) \leq A_{x} \exp \left(\frac{R}{C}\right) \quad \text { for all } R>0,
$$

where $C$ is the first sharpness constant of $\Gamma$.

The key idea of Theorem 13 is to bring branching laws to spectral analysis [10, 12], namely, we consider the restriction of irreducible representations of $G$ that are realized in the space of functions on the homogeneous space $X=G / H$ and analyze the $G$-representations when restricted to the subgroup $L$. Details will be given in [7]. 
Acknowledgements This article is based on the talk that the author delivered at the eleventh International Workshop: Lie Theory and its Applications in Physics in Varna, Bulgaria, 15-21, June, 2015. The author is grateful to Professor Vladimir Dobrev for his warm hospitality.

\section{References}

1. P. Buser, G. Courtois, Finite parts of the spectrum of a Riemann surface, Math. Ann. 287 (1990), pp. 523-530.

2. E. Calabi, L. Markus, Relativistic space forms, Ann. of Math. 75, (1962), pp. 63-76,

3. W. M. Goldman, Nonstandard Lorentz space forms, J. Differential Geom. 21 (1985), pp. 301-308.

4. F. Kassel, Deformation of proper actions on reductive homogeneous spaces, Math. Ann. 353, (2012), pp. 599-632.

5. F. Kassel, T. Kobayashi, Stable spectrum for pseudo-Riemannian locally symmetric spaces, C. R. Acad. Sci. Paris 349, (2011), pp. 29-33

6. F. Kassel, T. Kobayashi, Poincaré series for non-Riemannian locally symmetric spaces. Adv. Math. 287, (2016), pp. 123-236

7. F. Kassel, T. Kobayashi, Spectral analysis on standard non-Riemannian locally symmetric spaces, in preparation.

8. T. Kobayashi, Proper action on a homogeneous space of reductive type, Math. Ann. 285, (1989), pp. 249-263

9. T. Kobayashi, On discontinuous groups acting on homogeneous spaces with noncompact isotropy subgroups, J. Geom. Phys. 12, (1993), pp. 133-144

10. T. Kobayashi, Discrete decomposability of the restriction of $A_{\mathfrak{q}}(\lambda)$ with respect to reductive subgroups and its applications. Invent. Math. 117, (1994), pp. 181-205

11. T. Kobayashi, Deformation of compact Clifford-Klein forms of indefinite-Riemannian homogeneous manifolds, Math. Ann. 310, (1998), pp. 395-409

12. T. Kobayashi, Hidden symmetries and spectrum of the Laplacian on an indefinite Riemannian manifold, In: Spectral analysis in geometry and number theory, pp. 73-87. Contemporary Mathematics 484, Amer. Math. Soc., 2009.

13. T. Kobayashi, T. Yoshino, Compact Clifford-Klein forms of symmetric spaces - revisited, Pure Appl. Math. Q. 1, (2005), pp. 591-653

14. G. Margulis, Problems and conjectures in rigidity theory. In: Mathematics: Frontiers and Perspectives, pp. 161-174, Amer. Math. Soc., Providence, RI, 2000.

15. C. C. Moore, Ergodicity of flows on homogeneous spaces, Amer. J. Math. 88 (1966), 154178.

16. T. Okuda, Classification of semisimple symmetric spaces with proper $S L_{2}(\mathbb{R})$-actions, J. Differential Geom. 94 (2013), pp.301-342.

17. A. Oppenheim, (1929). The minima of indefinite quaternary quadratic forms". Proc. Nat. Acad. Sci. U.S.A. 15, (1929), pp. 724-727.

18. A. Weil, On discrete subgroups of Lie groups II, Ann. of Math. 75 (1962), pp. 578-602.

19. J. A. Wolf, Spaces of Constant Curvature, Sixth edition. AMS Chelsea Publishing, Providence, RI, 2011. xviii+424

20. S. A. Wolpert, Disappearance of cusp forms in special families, Ann. of Math. 139, (1994), 239-291. 